\title{
Adenocarcinoma of the penis
}

INSERM

\section{Source}

INSERM. (1999). Orphanet: an online rare disease and orphan drug data base.

Adenocarcinoma of the penis. ORPHA:398053

Adenocarcinoma of the penis is an extremely rare penile epithelial neoplasm,

histologically composed of nests of epithelilal cells floating in lakes of extracellular, PAS-

positive mucin, clinically characterized by a nonhealing ulcer or soft mass in the preputium or glans area, with itching and burning often preceding appearance of the lesion.

Lymphadenopathy may indicate dissemination. Mucinous metaplasia of the penis may be a risk factor. 